MATHEMATICS OF COMPUTATION

Volume 82, Number 283, July 2013, Pages 1379-1400

S 0025-5718(2013)02670-1

Article electronically published on February 20, 2013

\title{
A MIMETIC DISCRETIZATION OF ELLIPTIC OBSTACLE PROBLEMS
}

\author{
PAOLA F. ANTONIETTI, LOURENCO BEIRÃO DA VEIGA, AND MARCO VERANI
}

\begin{abstract}
We develop a Finite Element Method (FEM) which can adopt very general meshes with polygonal elements for the numerical approximation of elliptic obstacle problems. These kinds of methods are also known as mimetic discretization schemes, which stem from the Mimetic Finite Difference (MFD) method. The first-order convergence estimate in a suitable (mesh-dependent) energy norm is established. Numerical experiments confirming the theoretical results are also presented.
\end{abstract}

\section{INTRODUCTION}

Elliptic obstacle problems refer to find the equilibrium position of an elastic membrane whose boundary is held fixed, and which is constrained to lie above a given obstacle. It can be considered as a model problem for variational inequalities (see, e.g, 20]), and it has found applications in a number of different fields such as elasticity and fluid dynamics. For example, applications include fluid filtration in porous media, optimal control, and financial mathematics [23, 22].

In the present paper we develop, for the obstacle problem, a low order Finite Element Method (FEM) which can adopt very general meshes. These kinds of meshes are made of (possibly nonconvex) polygons of a variable number of edges, and do not have to fulfill matching conditions. These types of schemes, which stem from the Mimetic Finite Difference (MFD) method, are today known as mimetic discretization methods. The first papers introducing an interpretation of the Mimetic Finite Difference method as a generalization of the Finite Element Method are very recent [11, 13. Lately, this generalization of FEM has been applied to a wide range of problems, a very short list including $[9,12,4,14,3,25,5,2,24$.

The rest of the paper is organized as follows. In Section 2 we introduce the model problem, and fix some notation. The proposed method is introduced in Section 3 , and the convergence analysis is provided in Section 4. Finally, in Section 5 we discuss some implementation issues, and in Section [6 we show some numerical results.

Received by the editor May 10, 2010 and, in revised form, April 26, 2011.

2010 Mathematics Subject Classification. Primary 65N30; Secondary 35R35.

Key words and phrases. Mimetic Finite Difference Methods, obstacle problems.

The first and the third authors were supported in part by the Italian research project PRIN 2008: "Analysis and development of advanced numerical methods for PDEs". 


\section{The obstacle PRoblem}

Throughout the paper we will use standard notation for Sobolev spaces, norms and seminorms. For a bounded domain $D$ in $\mathbb{R}^{2}$, we denote by $H^{s}(D)$ the standard Sobolev space of order $s \geq 0$, and by $\|\cdot\|_{H^{s}(D)}$ and $|\cdot|_{H^{s}(D)}$ the usual Sobolev norm and seminorm, respectively. For $s=0$, we write $L^{2}(D)$ in lieu of $H^{0}(D) . H_{0}^{1}(D)$ is the subspace of $H^{1}(D)$ of functions with zero trace on $\partial D$.

Let $\Omega$ be an open, bounded, convex set of $\mathbb{R}^{2}$, with either a polygonal or a $C^{2}$-smooth boundary $\Gamma:=\partial \Omega$. Let $g:=\tilde{g}_{\mid \Gamma}$, with $\tilde{g} \in H^{2}(\Omega)$ and we set

$$
V^{g}:=\left\{v \in H^{1}(\Omega): v=g \text { on } \Gamma\right\} .
$$

Let us introduce the bilinear form $a(u, v): H^{1}(\Omega) \times H^{1}(\Omega) \longrightarrow \mathbb{R}$ defined by

$$
a(u, v):=\int_{\Omega} \nabla u \cdot \nabla v \mathrm{~d} x
$$

and the linear functional $F(v): H^{1}(\Omega) \longrightarrow \mathbb{R}$ with

$$
F(v):=\int_{\Omega} f v \mathrm{~d} x
$$

where we assume $f \in L^{2}(\Omega)$. Let us introduce the function $\psi \in H^{2}(\Omega)$ with $\psi \leq g$ on $\Gamma$ and the convex space

$$
K:=\left\{v \in V^{g}: v \geq \psi \text { a.e. in } \Omega\right\} .
$$

We are interested in solving the following variational inequality:

$$
\left\{\begin{array}{l}
\text { Find } u \in K \text { such that } \\
a(u, v-u) \geq F(v-u) \quad \forall v \in K .
\end{array}\right.
$$

It is well known (see e.g. 8] and [27, Corollary 5:2.3] ) that under the above data regularity assumption, the elliptic obstacle problem (2.1) admits a unique solution $u \in H^{2}(\Omega)$.

\section{A mimetic Discretization}

In this section we present a mimetic discretization method for the obstacle problem (2.1). This method is the direct extension of the scheme presented in 9] for the problem without obstacle.

3.1. Mesh notation and assumptions. Let $\Omega_{h} \subset \Omega$ be a polygonal approximation of $\Omega$, in such a way that all vertices of $\Omega_{h}$ which are on the boundary of $\Omega_{h}$ are also on the boundary of $\Omega$. The polygonal domain $\Omega_{h}$ represents the computational domain for the method. With a little abuse of notation, we also denote by $\Omega_{h}$ a partition of the above introduced computational domain into polygons $E$. We assume that this partition is conformal, i.e., an intersection of two different elements $E_{1}$ and $E_{2}$ is either a few mesh points, or a few mesh edges (two adjacent elements may share more than one edge) or empty. We allow $\Omega_{h}$ to contain nonconvex elements. Note, moreover, that, differently from conforming finite element meshes, T-junctions are now allowed in the mesh; indeed, this are included in the above conditions simply by splitting single edges into two new (aligned) edges. For each polygon $E, k_{E}$ denotes its number of vertexes, $|E|$ denotes its area, $h_{E}$ denotes its diameter and

$$
h:=\max _{E \in \Omega_{h}} h_{E} .
$$


We denote the set of mesh vertices and edges by $\mathcal{N}_{h}$ and $\mathcal{E}_{h}$, the set of internal vertices and edges by $\mathcal{N}_{h}^{0}$ and $\mathcal{E}_{h}^{0}$, the set of boundary vertices and edges by $\mathcal{N}_{h}^{\partial}$ and $\mathcal{E}_{h}^{\partial}$. The set of vertices and edges of a particular element $E$ are denoted by $\mathcal{N}_{h}^{E}$ and $\mathcal{E}_{h}^{E}$, respectively. Moreover, we denote a generic mesh vertex by v, a generic edge by e and its length both by $h_{\mathrm{e}}$ and $|\mathrm{e}|$. A fixed orientation is also set for the mesh $\Omega_{h}$, which is reflected by a unit normal vector $\mathbf{n}_{\mathrm{e}}$, e $\in \mathcal{E}_{h}$, fixed once for all. For every polygon $E$ and edge e $\in \mathcal{E}_{h}^{E}$, we define a unit normal vector $\mathbf{n}_{E}^{\mathrm{e}}$ that points outside $E$.

The mesh is assumed to satisfy the following shape regularity properties, which have already been used in [9]. There exist

- an integer number $N_{s}$ independent of $h$;

- a real positive number $\rho$ independent of $h$;

- a compatible sub-decomposition $\mathcal{T}_{h}$ of every $\Omega_{h}$ into shape-regular triangles, such that

(H1) any polygon $E \in \Omega_{h}$ admits a decomposition $\left.\mathcal{T}_{h}\right|_{E}$ formed by less than $N_{s}$ triangles;

(H2) any triangle $T \in \mathcal{T}_{h}$ is shape-regular in the sense that the ratio between the radius $r_{T}$ of the inscribed ball and the diameter $h_{T}$ of $T$ is bounded from below by $\rho$ :

$$
0<\rho \leq \frac{r_{T}}{h_{T}}
$$

From $(\mathrm{H} 1),(\mathrm{H} 2)$ there can be easily derived the following useful properties that we list below.

(M1) The number of vertexes and edges of every polygon $E$ of $\Omega_{h}$ are uniformly bounded from above by two integer numbers $N_{\mathrm{v}}$ and $N_{\mathrm{e}}$, which only depend on $N_{s}$.

(M2) There exists a real positive number $\sigma_{s}$, which only depends on $N_{s}$ and $\rho$, such that

$$
h_{\mathrm{e}} \geq \sigma_{s} h_{E} \quad \text { and } \quad|E| \geq \sigma_{s} h_{E}^{2},
$$

for every polygon $E$ of every decomposition $\Omega_{h}$, and for every edge e of $E$.

(M3) There exists a constant $C_{a}$, only dependent on $\rho$ and $N_{s}$, such that for every polygon $E$, for every edge e of $E$ and for every function $\psi \in H^{1}(E)$ the following trace inequality holds:

$$
\|\psi\|_{L^{2}(\mathrm{e})}^{2} \leq C_{a}\left(h_{E}^{-1}\|\psi\|_{L^{2}(E)}^{2}+h_{E}|\psi|_{H^{1}(E)}^{2}\right) .
$$

(M4) There exists a constant $C_{a p p}$, which is independent of $h$, such that the following holds. For every $E$ and for every function $\psi \in H^{m}(E), m \in \mathbb{N}$, there exists a polynomial $\psi_{k}$ of degree $k$ living on $E$ such that

$$
\left|\psi-\psi_{k}\right|_{H^{l}(E)} \leq C_{a p p} h_{E}^{m-l}|\psi|_{H^{m}(E)}
$$

for all integers $0 \leq l \leq m \leq k+1$.

Since the polygons $E$ may be nonstar-shaped, we show for completeness the proof of (M4) in the Appendix. 
3.2. Degrees of freedom and interpolation operators. The discretization of problem (2.1) requires to discretize a scalar field in $H^{1}(\Omega)$. In order to do so, we start introducing the degrees of freedom for the discrete approximation space. The discrete space $V_{h}$ is defined as follows: a vector $v_{h} \in V_{h}$ consists of a collection of degrees of freedom

$$
v_{h}:=\left\{v^{\vee}\right\}_{\mathrm{v} \in \mathcal{N}_{h}},
$$

one per mesh vertex, e.g., to every vertex $\vee \in \mathcal{N}_{h}$, we associate a real number $v^{v}$. The scalar $v^{v}$ represents the nodal value of the underlying discrete scalar field of displacement. The number of unknowns is equal to the number of vertices of the mesh. We also define the discrete space $V_{h}^{g} \subset V_{h}$ of functions which satisfy the Dirichlet boundary conditions

$$
V_{h}^{g}:=\left\{v_{h} \in V_{h}: v_{h}^{v}=g(\mathrm{v}) \forall \mathrm{v} \in \mathcal{N}_{h}^{\partial}\right\} .
$$

Accordingly, $V_{h}^{0}$ represents the space of discrete functions which vanish at the boundary nodes.

We define the following interpolation operator from the spaces of smooth enough functions to the discrete space $V_{h}$. For every function $v \in \mathcal{C}^{0}(\bar{\Omega}) \cap H^{1}(\Omega)$, we define $v_{\mathrm{I}} \in V_{h}$ by

$$
v_{\mathrm{I}}^{\mathrm{v}}:=v(\mathrm{v}) \quad \forall \mathrm{v} \in \mathcal{N}_{h} .
$$

Moreover, we analogously define the local interpolation operator from $\mathcal{C}^{0}(\bar{E}) \cap$ $H^{1}(E)$ into $\left.V_{h}\right|_{E}$ given by

$$
v_{\mathrm{I}}^{\mathrm{v}}:=v(\mathrm{v}) \quad \forall \mathrm{v} \in \mathcal{N}_{h}^{E} .
$$

3.3. Discrete norms and bilinear forms. We endow the space $V_{h}$ with the discrete seminorm

$$
\left\|v_{h}\right\|_{1, h}^{2}:=\sum_{E \in \Omega_{h}}\left\|v_{h}\right\|_{1, h, E}^{2}=\sum_{E \in \Omega_{h}}|E| \sum_{\mathrm{e} \in \mathcal{E}_{h}^{E}}\left[\frac{1}{|\mathrm{e}|}\left(v^{\mathrm{v}_{2}}-v^{\mathrm{v}_{1}}\right)\right]^{2},
$$

where $\mathrm{v}_{1}$ and $\mathrm{v}_{2}$ are the two vertexes of e. The quantity $\|\cdot\|_{1, h}$ is a $H^{1}(\Omega)$ type discrete seminorm, which becomes a norm on $V_{h}^{0}$. Indeed, the differences $\frac{1}{|\mathrm{e}|}\left(v^{\mathrm{v}_{2}}-v^{\mathrm{v}_{1}}\right)$ represent gradients on edges and the scalings with respect to $h_{E}$ and $h_{\mathrm{e}}$ are the correct ones to mimic an $H^{1}(E)$ local seminorm. In the numerical tests we will also consider the following $L^{2}(\Omega)$ - and $L^{\infty}(\Omega)$-type discrete norms

$$
\begin{aligned}
\left\|v_{h}\right\|_{0, h}^{2} & :=\sum_{E \in \Omega_{h}}|E| \sum_{\mathrm{v} \in \mathcal{N}_{h}^{E}}\left(v^{v}\right)^{2}, \\
\left\|v_{h}\right\|_{\infty, h} & :=\max _{\mathrm{v} \in \mathcal{N}_{h}}\left|v^{\mathrm{v}}\right| .
\end{aligned}
$$

We denote by $a_{h}(\cdot, \cdot): V_{h} \times V_{h} \rightarrow \mathbb{R}$ the discretization of the bilinear form $a(\cdot, \cdot)$, defined as

$$
a_{h}\left(v_{h}, w_{h}\right):=\sum_{E \in \Omega_{h}} a_{h}^{E}\left(v_{h}, w_{h}\right) \quad \forall v_{h}, w_{h} \in V_{h},
$$

where $a_{h}^{E}(\cdot, \cdot)$ is a symmetric bilinear form on each element $E$. The local forms mimick

$$
a_{h}^{E}\left(v_{h}, w_{h}\right) \sim \int_{E} \nabla \widetilde{v}_{h} \cdot \nabla \widetilde{w}_{h} \mathrm{~d} x
$$

where, roughly speaking, $\widetilde{v}_{h}, \widetilde{w}_{h}$ denote regular functions living on $E$ which "extend the data" $v_{h}, w_{h}$ inside the element. 
We introduce two fundamental assumptions for the local bilinear form $a_{h}^{E}(\cdot, \cdot)$. The first one represents the coercivity (up to the kernel) and the correct scaling with respect to the element size.

(S1) There exist two positive constants $c_{1}$ and $c_{2}$ independent of $h$ such that, for every $u_{h}, v_{h} \in V_{h}$ and each $E \in \Omega_{h}$, we have

$$
c_{1}\left\|v_{h}\right\|_{1, h, E}^{2} \leq a_{h}^{E}\left(v_{h}, v_{h}\right), \quad \quad a_{h}^{E}\left(u_{h}, v_{h}\right) \leq c_{2}\left\|u_{h}\right\|_{1, h, E}\left\|v_{h}\right\|_{1, h, E} .
$$

In order to introduce the second assumption, we observe beforehand that, using an integration by parts,

$$
\begin{aligned}
\int_{E} \nabla v \cdot \nabla p^{1} \mathrm{~d} x & =-\int_{E}\left(\Delta p^{1}\right) v \mathrm{~d} x+\sum_{\mathrm{e} \in \mathcal{E}_{h}^{E}} \int_{\mathrm{e}}\left(\nabla p^{1} \cdot \mathbf{n}_{E}^{\mathrm{e}}\right) v \mathrm{~d} s \\
& =\sum_{\mathrm{e} \in \mathcal{E}_{h}^{E}} \nabla p^{1} \cdot \mathbf{n}_{E}^{\mathrm{e}} \int_{\mathrm{e}} v \mathrm{~d} s
\end{aligned}
$$

for all $E \in \Omega_{h}$, for all $v \in\left[H^{1}(E)\right]^{2}$ and for all linear functions $p^{1}$. By substituting the integral in the last term of (3.5) with a trapezium integration rule gives our second condition.

(S2) For every element $E$, every linear vector function $p^{1}$ on $E$, and every $v_{h} \in$ $V_{h}$, it holds that

$$
a_{h}^{E}\left(v_{h},\left(p^{1}\right)_{\mathrm{I}}\right)=\sum_{\mathrm{e} \in \mathcal{E}_{h}^{E}}\left(\nabla p^{1} \cdot \mathbf{n}_{E}^{\mathrm{e}}\right) \frac{|\mathrm{e}|}{2}\left(v_{h}^{\mathrm{v}_{1}}+v_{h}^{\mathrm{v}_{2}}\right),
$$

where $\mathbf{v}_{1}$ and $\mathbf{v}_{2}$ are the two vertices of $\mathrm{e} \in \mathbf{n}_{E}^{\mathrm{e}}$.

The meaning of the above consistency condition (S2) is therefore that the discrete bilinear form respects integration by parts when tested with linear functions.

Remark 3.1. The scalar product and the bilinear form shown in this section can be easily built element by element in a simple algebraic way. A brief description of such construction can be found in Section 5]

3.4. The discrete method. Finally, we are able to define the proposed mimetic discrete method for the obstacle problem. Let the loading term

$$
\left(f, v_{h}\right)_{h}:=\left.\sum_{E \in \Omega_{h}} \bar{f}\right|_{E} \sum_{i=1}^{k_{E}} v^{v_{i}} \omega_{E}^{i}
$$

where $\mathrm{v}_{1}, \ldots, \mathrm{v}_{k_{E}}$ are the vertices of $E,\left.\bar{f}\right|_{E}:=\frac{1}{|E|} \int_{E} f \mathrm{~d} x$, and $\omega_{E}^{1}, \ldots, \omega_{E}^{k_{E}}$ are positive weights such that $\sum_{i=1}^{k_{E}} \omega_{E}^{i}=|E|$. The above loading term is an approximation of

$$
\left(f, v_{h}\right)_{h} \sim \int_{\Omega} f \tilde{v}_{h} \mathrm{~d} x
$$

which is exact for constant functions.

Let us introduce the convex space

$$
K_{h}:=\left\{v_{h} \in V_{h}^{g}: v_{h}^{v} \geq \psi(\mathrm{v}) \forall \mathrm{v} \in \mathcal{N}_{h}\right\} .
$$

Then, the mimetic discretization of problem (2.1) reads:

$$
\left\{\begin{array}{l}
\text { Find } u_{h} \in K_{h} \text { such that } \\
a_{h}\left(u_{h}, v_{h}-u_{h}\right) \geq\left(f, v_{h}-u_{h}\right)_{h} \quad \forall v_{h} \in K_{h} .
\end{array}\right.
$$


Due to property (S1) it is immediate to check that the bilinear form $a_{h}(\cdot, \cdot)$ is coercive on $V_{h} / \mathbb{R}$. As a consequence, recalling again that $K_{h} \subset V_{h}$ is convex and closed, standard results [15] give the existence and uniqueness of a solution for the discrete problem (3.8). The uniform stability of the problem with respect to $h$ will be left as an implicit consequence of the analysis that follows.

\section{Convergence of the method}

In this section we prove the linear convergence of the proposed discrete method. In the following, we will use the symbols $\simeq, \lesssim, \gtrsim$ to represent equivalences and bounds which hold up to a constant independent of the mesh-size.

4.1. A lifting operator. In this section we show that, for all $E \in \Omega_{h}$, there exists a suitable lifting operator

$$
R_{h}^{E}:\left.V_{h}\right|_{E} \longrightarrow H^{1}(E) \cap \mathcal{C}^{0}(\bar{E}),
$$

which satisfies the following properties.

(L1) $\left(R_{h}^{E} v_{h}\right)(\mathrm{v})=v_{h}^{\vee} \quad \forall \mathrm{v} \in \mathcal{N}_{h}^{E}$ and $\left.\forall v_{h} \in V_{h}\right|_{E}$;

(L2) $\left.R_{h}^{E} v_{h}\right|_{\mathrm{e}}$ is a linear function $\forall \mathrm{e} \in \mathcal{E}_{h}^{E}$ and $\left.\forall v_{h} \in V_{h}\right|_{E}$;

(L3) $R_{h}^{E}\left(p^{1}\right)_{\mathrm{I}}=p^{1}$ for all linear functions $p^{1}$ on $E$;

(L4) $\left.\left|R_{h}^{E} v_{h}\right|_{H^{1}(E)}^{2} \lesssim\left\|v_{h}\right\|_{1, h, E}^{2} \quad \forall v_{h} \in V_{h}\right|_{E}$;

(L5) $\left\|R_{h}^{E} v_{\mathrm{I}}\right\|_{L^{2}(E)}^{2} \lesssim \sum_{k=0}^{2} h_{E}^{2 k}|v|_{H^{k}(E)}^{2} \quad \forall v \in H^{2}(E)$;

(L6) The following maximum principle holds: for all $\left.v_{h} \in V_{h}\right|_{E}$, if $v_{h}^{\vee} \geq 0 \forall \mathrm{v} \in$ $\mathcal{N}_{h}^{E}$, then the lifting operator satisfies $R_{h}^{E} v_{h} \geq 0$ in $E$.

Note that, due to properties (L1) and (L2), the global lifting operator

$$
\begin{aligned}
& R_{h}: V_{h} \longrightarrow H^{1}\left(\Omega_{h}\right) \cap \mathcal{C}^{0}\left(\bar{\Omega}_{h}\right), \\
& \left.R_{h}\left(v_{h}\right)\right|_{E}:=R_{h}^{E}\left(\left.v_{h}\right|_{E}\right) \quad \forall v_{h} \in V_{h}, E \in \Omega_{h},
\end{aligned}
$$

is well defined.

The local lifting operator is built as in [6], which in turn is an improved version of that presented in [9]. Note that we cannot directly use the lifting operator of [9] since it does not preserve linear functions. Let $E \in \Omega_{h}$, for a given $\left.v_{h} \in V_{h}\right|_{E}$, the function $R_{h}^{E} v_{h}$ is globally continuous and piecewise linear on the sub-triangulation $\mathcal{T}_{h}$ and it is defined in the following way. On the vertices $\mathrm{v} \in \mathcal{N}_{h}^{E}$ we set $R_{h}^{E} v_{h}(\mathrm{v})=$ $v_{h}^{v}$. On the remaining nodes of $\mathcal{T}_{h}$ that lie on the boundary, $R_{h}^{E} v_{h}$ is defined by linear interpolation of the two vertex values of the edge. On the internal nodes of $E$, we do instead the following construction. Given any internal node $\vee$ of $\mathcal{T}_{h}$, we call $\Xi_{\mathrm{v}}$ the set of nodes which share an edge with $v$ and are different from $v$. Then, it is easy to check that $v$, which lies in the convex hull determined by the nodes $\{\bar{v}\}_{\bar{v} \in \Xi_{\mathrm{v}}}$, can be expressed (in a nonunique way) as a weighted sum

$$
\mathrm{v}=\sum_{\overline{\mathrm{v}} \in \Xi_{\mathrm{v}}} w_{\overline{\mathrm{v}}}^{\mathrm{v}} \overline{\mathrm{v}}
$$

with $w_{\bar{v}}^{v}$ nonnegative real numbers such that $\sum_{\bar{v} \in \Xi_{v}} w_{\bar{v}}^{v}=1$. For each internal node $\mathrm{v}$, we then enforce the condition

$$
R_{h}^{E} v_{h}(\mathrm{v})-\sum_{\overline{\mathrm{v}} \in \Xi_{\mathrm{v}}} w_{\overline{\mathrm{v}}}^{\mathrm{v}} R_{h}^{E} v_{h}(\overline{\mathrm{v}})=0 .
$$

This set of conditions provides a square linear system which determines the value of $R_{h}^{E} v_{h}$ in the internal nodes. Indeed, it is immediate to verify that the associated 
matrix is an M-matrix, which in particular implies the existence of a unique solution and a discrete maximum principle. Therefore, assumption (L6) is satisfied.

Properties (L1) and (L2) are clearly satisfied by construction. Furthermore, following the same argument as in 9], from the maximum principle it follows that the operator $R_{h}^{E}$ satisfies also the stability condition (L4). We now check property (L3). Let $p_{1}$ be a linear function on $E$. Since the solution of the linear system introduced above is unique, in order to show $R_{h}^{E}\left(p^{1}\right)_{\mathrm{I}}=p^{1}$ it is sufficient to prove that $p_{1}$ satisfies (4.2) for any $v$ internal node of $E$. Using (4.1) and recalling that $p_{1}$ is linear, it holds that

$$
p_{1}(\mathrm{v})=p_{1}\left(\sum_{\overline{\mathrm{v}} \in \Xi_{\mathrm{v}}} w_{\overline{\mathrm{v}}}^{\vee} \overline{\mathrm{v}}\right)=\sum_{\overline{\mathrm{v}} \in \Xi_{\mathrm{v}}} w_{\overline{\mathrm{v}}}^{\vee} p_{1}(\overline{\mathrm{v}})
$$

for all the internal nodes $v$ of $E$, which is exactly (4.2) for the function $p_{1}$.

We are left to show property (L5). We start observing that, due to the maximum principle,

$$
\left\|R_{h}^{E} v_{I}\right\|_{L^{\infty}(E)} \leq \max _{\mathrm{v} \in \mathcal{N}_{h}^{E}}\left|v_{I}^{\mathrm{v}}\right|=\max _{\mathrm{v} \in \mathcal{N}_{h}^{E}}|v(\mathrm{v})| \leq\|v\|_{L^{\infty}(E)} .
$$

Moreover, due to $(\mathrm{H} 1)$ and $(\mathrm{H} 2)$ it is easy to check that

$$
h_{E} \lesssim h_{T} \leq\left. h_{E} \quad \forall T \in \mathcal{T}_{h}\right|_{E} .
$$

Using (4.3), a scaling argument on each triangle of $\left.T \in \mathcal{T}_{h}\right|_{E}$, and finally (4.4), we get

$$
\begin{aligned}
\left\|R_{h}^{E} v_{I}\right\|_{L^{2}(E)}^{2} & \leq|E|\|v\|_{L^{\infty}(E)}^{2}=|E| \max _{T \in \mathcal{T}_{h}}\|v\|_{L^{\infty}(T)}^{2} \\
& \leq|E| \max _{T \in \mathcal{T}_{h}} \sum_{k=0}^{2} h_{T}^{2 k-2}|v|_{H^{k}(T)}^{2} \lesssim|E| \sum_{k=0}^{2} h_{E}^{2 k-2}|v|_{H^{k}(E)}^{2} .
\end{aligned}
$$

Property (L5) follows from the above bound and $|E| \leq h_{E}^{2}$. This completes the proof of properties (L1)-(L6).

Finally, we make the following two observations. Given any $E \in \Omega_{h}$ and $v \in$ $H^{2}(E)$, let $v_{1}$ be its linear approximation introduced in (M4) setting $k=1$. Then, using (L3) and (L5), and finally the approximation property (M4) we get

$$
\begin{aligned}
\left\|v-R_{h}^{E} v_{\mathrm{I}}\right\|_{L^{2}(E)}^{2} & \lesssim\left\|v-v_{1}\right\|_{L^{2}(E)}^{2}+\left\|R_{h}^{E}\left(v_{1}-v\right)_{\mathrm{I}}\right\|_{L^{2}(E)}^{2} \\
& \lesssim \sum_{k=0}^{2} h_{E}^{2 k}\left|v-v_{1}\right|_{H^{k}(E)}^{2} \lesssim h_{E}^{4}|v|_{H^{2}(E)}^{2}
\end{aligned}
$$

for all $E \in \Omega_{h}$. Furthermore, due to the maximum principle property (L6) and the definition (3.2) of discrete $H^{1}$-norm it follows that

$$
\left\|R_{h}^{E} v_{h}-v_{h}^{\vee}\right\|_{L^{\infty}(E)} \leq \max _{\mathrm{v}^{\prime} \in \mathcal{N}_{h}^{E}}\left|v_{h}^{\mathrm{v}^{\prime}}-v_{h}^{\mathrm{v}}\right| \lesssim\left\|v_{h}\right\|_{1, h}
$$

which also gives immediately

$$
\left\|R_{h}^{E} v_{h}-v_{h}^{\vee}\right\|_{L^{2}(E)} \lesssim h_{E}\left\|v_{h}\right\|_{1, h} .
$$


4.2. A convergence result. In this section, we prove a convergence result for the mimetic discretization method applied to the variational inequality (2.1). The proof takes the steps from [10] (see also Remark 4.2 below).

Theorem 4.1. Let $u \in K \cap H^{2}(\Omega)$ be the solution to the continuous problem (2.1), and $u_{h} \in K_{h}$ the corresponding mimetic approximation, obtained by solving the discrete problem (3.8). Then, it holds that

$$
\left\|u_{h}-u_{I}\right\|_{1, h} \leq C h,
$$

where the constant $C$ is independent of the mesh-size $h$.

Proof. We set $e_{h}:=u_{h}-u_{\mathrm{I}}$. Let $u^{1}$ be the piecewise discontinuous linear function defined on $\Omega_{h}$ as follows: for every $E \in \Omega_{h}, u^{1}{ }_{\mid E}$ is the $L^{2}(E)$-projection of $u$ over the space of polynomials of degree $\leq 1$. With a little abuse of notation, in the following we indicate with $\left(u^{1}\right)_{\text {I }}$ a collection of nodal values such that for all elements $E$ the restriction $\left.\left(u^{1}\right)_{I}\right|_{E}$ is given by the local interpolation $\left(\left.u^{1}\right|_{E}\right)_{I}$. Note that both the $\|\cdot\|_{1, h}$ norm and the bilinear form $a_{h}$ can be immediately extended to $\left(u^{1}\right)_{I}$, since both operators are a sum of local terms. We observe that, due to (L2), it holds that

$$
\frac{|\mathrm{e}|}{2}\left(e_{h}^{\mathrm{v}_{1}}+e_{h}^{\mathrm{v}_{2}}\right)=\int_{\mathrm{e}} R_{h}^{E} e_{h} \mathrm{~d} x \quad \forall E \in \Omega_{h}, \mathrm{e} \in \mathcal{E}_{h}^{E} .
$$

By using (S1)-(S2), the discrete problem (3.8), and the above observation we get

$$
\begin{aligned}
c_{1}\left\|e_{h}\right\|_{1, h}^{2} & \leq a_{h}\left(e_{h}, e_{h}\right) \\
& \leq\left(f, e_{h}\right)_{h}-a_{h}\left(u_{\mathrm{I}}, e_{h}\right) \\
& =\left(f, e_{h}\right)_{h}-a_{h}\left(u_{\mathrm{I}}-\left(u^{1}\right)_{\mathrm{I}}, e_{h}\right)-a_{h}\left(\left(u^{1}\right)_{\mathrm{I}}, e_{h}\right) \\
& \leq\left(f, e_{h}\right)_{h}+c_{2}\left\|u_{\mathrm{I}}-\left(u^{1}\right)_{\mathrm{I}}\right\|_{1, h}\left\|e_{h}\right\|_{1, h}-\sum_{E \in \Omega_{h}} \sum_{\mathrm{e} \in \mathcal{E}_{h}^{E}} \frac{\partial u^{1}}{\partial \mathbf{n}_{E}^{\mathrm{e}}} \int_{e} R_{h}^{E} e_{h} \mathrm{~d} x .
\end{aligned}
$$

From (4.8), using twice an integration by parts and that $R_{h} e_{h}$ vanishes on the boundary of $\Omega_{h}$, it follows that

$$
\begin{aligned}
c_{1}\left\|e_{h}\right\|_{1, h}^{2} \leq & \left(f, e_{h}\right)_{h}+c_{2}\left\|u_{\mathrm{I}}-\left(u^{1}\right)_{\mathrm{I}}\right\|_{1, h}\left\|e_{h}\right\|_{1, h}-\sum_{E \in \Omega_{h}} \int_{E} \nabla R_{h}^{E} e_{h} \cdot \nabla u^{1} \mathrm{~d} x \\
= & \left(f, e_{h}\right)_{h}+c_{2}\left\|u_{\mathrm{I}}-\left(u^{1}\right)_{\mathrm{I}}\right\|_{1, h}\left\|e_{h}\right\|_{1, h}+\sum_{E \in \Omega_{h}} \int_{E} \nabla R_{h}^{E} e_{h} \cdot \nabla\left(u-u^{1}\right) \mathrm{d} x \\
& -\sum_{E \in \Omega_{h}} \int_{E} \nabla R_{h}^{E} e_{h} \cdot \nabla u \mathrm{~d} x \\
= & \left(f, e_{h}\right)_{h}+c_{2}\left\|u_{\mathrm{I}}-\left(u^{1}\right)_{\mathrm{I}}\right\|_{1, h}\left\|e_{h}\right\|_{1, h}+\sum_{E \in \Omega_{h}} \int_{E} \nabla R_{h}^{E} e_{h} \cdot \nabla\left(u-u^{1}\right) \mathrm{d} x \\
& +\int_{\Omega_{h}} \Delta u R_{h}^{E} e_{h} \mathrm{~d} x .
\end{aligned}
$$

Let us first estimate the term $\left\|u_{\mathrm{I}}-\left(u^{1}\right)_{\mathrm{I}}\right\|_{1, h} \equiv\left\|\left(u-u^{1}\right)_{\mathrm{I}}\right\|_{1, h}$. For simplicity, we set $v=u-u^{1}$. Using definition (3.2) of the norm $\|\cdot\|_{1, h}$ and the Cauchy-Schwarz 
inequality, we get

$$
\begin{aligned}
\left\|v_{\mathrm{I}}\right\|_{1, h}^{2} & =\sum_{E \in \Omega_{h}}|E| \sum_{\mathrm{e} \in \mathcal{E}_{h}^{E}}\left[\frac{1}{|\mathrm{e}|}\left(v^{\mathrm{v}_{2}}-v^{\mathrm{v}_{1}}\right)\right]^{2}=\sum_{E \in \Omega_{h}}|E| \sum_{\mathrm{e} \in \mathcal{E}_{h}^{E}}\left[\frac{1}{|\mathrm{e}|} \int_{e} v^{\prime} \mathrm{d} s\right]^{2} \\
& \leq \sum_{E \in \Omega_{h}}|E| \sum_{\mathrm{e} \in \mathcal{E}_{h}^{E}}\left[\frac{1}{|\mathrm{e}|}\|\nabla v\|_{L^{2}(\mathrm{e})}^{2}\right] .
\end{aligned}
$$

Applying the trace inequality (3.1) to $\nabla v$ and employing a standard interpolation error estimate yield

$$
\left\|\left(u-u^{1}\right)_{\mathrm{I}}\right\|_{1, h}^{2} \lesssim \sum_{E \in \Omega_{h}}\left[\left\|\nabla\left(u-u^{1}\right)\right\|_{L^{2}(E)}^{2}+h_{E}^{2}|u|_{H^{2}(E)}^{2}\right] \lesssim h^{2}|u|_{H^{2}(\Omega)}^{2} .
$$

From (4.9), by employing the Young inequality combined with (4.10) and introducing $w=\Delta u+f$, we get

$$
\begin{aligned}
\left\|e_{h}\right\|_{1, h}^{2} & \lesssim\left\{\left(f, e_{h}\right)_{h}-\int_{\Omega_{h}} f R_{h} e_{h} \mathrm{~d} x\right\}+h^{2}|u|_{H^{2}(\Omega)}^{2} \\
& +\sum_{E \in \Omega_{h}} \int_{E} \nabla R_{h}^{E} e_{h} \cdot \nabla\left(u-u^{1}\right) \mathrm{d} x+\int_{\Omega_{h}} w R_{h}^{E} e_{h} \mathrm{~d} x .
\end{aligned}
$$

As shown in [7], it holds that

$$
w \leq 0 \quad \text { and } \quad w(\psi-u)=0 \quad \text { a.e. in } \Omega_{h} .
$$

Simply adding and subtracting terms, we obtain

$$
\begin{aligned}
\int_{\Omega_{h}} w R_{h}^{E} e_{h} \mathrm{~d} x= & -\int_{\Omega_{h}} w\left(R_{h}^{E} u_{\mathrm{I}}-u\right) \mathrm{d} x+\int_{\Omega_{h}} w(\psi-u) \mathrm{d} x \\
& +\int_{\Omega_{h}} w\left(R_{h}^{E} u_{h}-R_{h}^{E} \psi_{\mathrm{I}}\right) \mathrm{d} x+\int_{\Omega_{h}} w\left(R_{h}^{E} \psi_{\mathrm{I}}-\psi\right) \mathrm{d} x .
\end{aligned}
$$

The second term in the right-hand side of (4.14) vanishes due to (4.13). Moreover, as for every $\mathrm{v} \in \mathcal{N}_{h}$ there holds $u_{h}(\mathrm{v}) \geq \psi_{\mathrm{I}}(\mathrm{v})$, employing assumption (L6), yields

$$
R_{h}^{E} u_{h}-R_{h}^{E} \psi_{\mathrm{I}} \geq 0 \quad \text { in } \Omega_{h},
$$

which recalling (4.13) gives $\int_{\Omega_{h}} w\left(R_{h}^{E} u_{h}-R_{h}^{E} \psi_{\mathrm{I}}\right) \mathrm{d} x \leq 0$. Hence, combining these last two observations with (4.14), we get

$$
\int_{\Omega_{h}} w R_{h}^{E} e_{h} \mathrm{~d} x \leq \int_{\Omega_{h}} w\left(u-R_{h}^{E} u_{\mathrm{I}}\right) \mathrm{d} x+\int_{\Omega_{h}} w\left(R_{h}^{E} \psi_{\mathrm{I}}-\psi\right) \mathrm{d} x .
$$

The above bound, using the Cauchy-Schwarz inequality, (4.6), and recalling that $w=\Delta u+f$, yields

$$
\int_{\Omega_{h}} w R_{h}^{E} e_{h} \mathrm{~d} x \lesssim h^{2}\|w\|_{L^{2}\left(\Omega_{h}\right)}\left(|\psi|_{H^{2}\left(\Omega_{h}\right)}+|u|_{H^{2}\left(\Omega_{h}\right)}\right) \lesssim h^{2} .
$$

We now estimate the remaining pieces in (4.11). By using (4.7) and proceeding as in the estimate of the First Piece in [9], it is easy to check that the following holds:

$$
\left|\left(f, e_{h}\right)_{h}-\left(f, R_{h}^{E} e_{h}\right)\right| \lesssim h\|f\|_{L^{2}(\Omega)}\left\|e_{h}\right\|_{1, h} \lesssim h\left\|e_{h}\right\|_{1, h} .
$$


Using the Cauchy-Schwarz inequality, assumption (L4), and a standard interpolation error estimate, yields

$$
\begin{aligned}
\sum_{E \in \Omega_{h}} \int_{E} \nabla R_{h}^{E} e_{h} \cdot \nabla\left(u-u^{1}\right) \mathrm{d} x & \leq\left\|\nabla R_{h}^{E} e_{h}\right\|_{L^{2}\left(\Omega_{h}\right)}\left\|\nabla\left(u-u^{1}\right)\right\|_{L^{2}\left(\Omega_{h}\right)} \\
& \lesssim h\left\|e_{h}\right\|_{1, h}|u|_{H^{2}} \lesssim h\left\|e_{h}\right\|_{1, h} .
\end{aligned}
$$

Combining (4.11) with (4.15), (4.16) and (4.17) finally gives

$$
\left\|e_{h}\right\|_{1, h}^{2} \lesssim h\left\|e_{h}\right\|_{1, h}+h^{2},
$$

which immediately gives the result.

Remark 4.2. We remark that the standard finite element techniques employed in [10] to prove a similar result cannot be directly applied in our context. Indeed, in the MFD framework the continuous and the discrete solutions are of a different nature: the continuous solution is a function, whereas the discrete approximation is a vector. As a consequence, the continuous and the discrete problems cannot be simply summed up to obtain Céa-like results, as in Theorem 2.1 in [10, which is a crucial step to derive the convergence result in the finite element context.

Remark 4.3. The convexity condition on $\Omega$ can be relaxed to include a more general class of domains, provided that the solution $u$ still belongs to $H^{2}(\Omega)$, and $\Omega_{h}$ can be inscribed in $\Omega$ for every $h$ (e.g., nonconvex polygonal domains). Indeed, following the same argument as in [10, which is based on a suitable extension of the mesh and solution, the convergence result can be easily extended.

Remark 4.4. Whenever $V^{g}$ coincide with $H_{0}^{1}(\Omega)$, i.e., homogeneous boundary conditions are imposed on the domain boundary, Theorem 4.1 can be proved also in a different way, following the idea proposed in [19]. We refer to [1, Appendix A] for the details.

\section{IMPLEMENTATION ISSUES}

In this section we show briefly how the local scalar product appearing in (3.4) is built in practice. Let $E$ be a general element of $\Omega_{h}$, with $k_{E} \geq 3$ vertices. Then, we need to build an $k_{E} \times k_{E}$ symmetric matrix $\mathrm{M}$ which represents the local scalar product

$$
a_{h}^{E}\left(v_{h}, w_{h}\right)=v_{h}^{T} \mathrm{M} w_{h} \quad \forall v_{h}, w_{h} \in \mathbb{R}^{k_{E}} .
$$

Let the functions $\rho_{1}:=1, \rho_{2}:=x-\bar{x}, \rho_{3}:=y-\bar{y}$ represent a basis for the space of the linear polynomials on $E$, with $x, y$ cartesian coordinates in the plane and $(\bar{x}, \bar{y})$ representing the position of the center of mass of $E$. Then, we introduce the $k_{E} \times 3$ matrix $\mathrm{N}$ given by

$$
\mathrm{N}(i, j):=\rho_{j}\left(\mathrm{v}_{i}\right) \quad i=1, \ldots, k_{E}, j=1,2,3,
$$

where $\mathbf{v}_{1}=\left(x_{1}, y_{1}\right), \ldots, \mathbf{v}_{k_{E}}=\left(x_{k_{E}}, y_{k_{E}}\right)$ are the $k_{E}$ vertices of the polygon $E$, i.e.,

$$
\mathrm{N}:=\left(\begin{array}{ccc}
1 & x_{1}-\bar{x} & y_{1}-\bar{y} \\
1 & x_{2}-\bar{x} & y_{2}-\bar{y} \\
1 & x_{3}-\bar{x} & y_{3}-\bar{y} \\
\vdots & \vdots & \\
1 & x_{k_{E}}-\bar{x} & y_{k_{E}}-\bar{y}
\end{array}\right) .
$$


Then, it is easy to check that the consistency condition (S2) can be expressed as

$$
v_{h}^{T} \mathrm{MN}=v_{h}^{T} \mathrm{R} \quad \forall v_{h} \in \mathbb{R}^{k_{E}},
$$

where the $k_{E} \times 3$ matrix $\mathbb{R}$ with columns $\mathrm{R}_{\mid j}, j=1,2,3$, is the unique matrix that represents the right-hand side of (S2)

$$
v_{h}^{T} \mathrm{R}_{\mid j}=\sum_{\mathrm{e} \in \mathcal{E}_{h}^{E}}\left(\nabla \rho_{j} \cdot \mathbf{n}_{E}^{\mathrm{e}}\right) \frac{|\mathrm{e}|}{2}\left(v_{h}^{\mathrm{v}_{1}}+v_{h}^{\mathrm{v}_{2}}\right) \quad \forall v_{h} \in \mathbb{R}^{k_{E}} .
$$

More precisely, for $i=1, \ldots, k_{E}$, let $\mathrm{e}_{i}$ be the edge connecting the vertexes $\mathrm{v}_{i}=$ $\left(x_{i}, y_{i}\right)$ and $\mathrm{v}_{i+1}=\left(x_{i+1}, y_{i+1}\right)$ (with the convention that $\left.\mathrm{v}_{k_{E}+1} \equiv \mathrm{v}_{1}\right)$, and let $\mathbf{n}_{E}^{\mathbf{e}_{i}} \in \mathbf{R}^{1 \times 2}$ be the corresponding outward normal vector. Clearly, $\left|\mathrm{e}_{i}\right| \mathbf{n}_{E}^{\mathrm{e}_{i}}=\left(y_{i+1}-\right.$ $\left.y_{i}, x_{i}-x_{i+1}\right)$. Therefore, the matrix $\mathrm{R}$ has the following form:

$$
\begin{aligned}
\mathbf{R} & =\left(\begin{array}{ccc}
0 & \left(\left|\mathrm{e}_{k_{E}}\right| \mathbf{n}_{E}^{\mathrm{e}_{k_{E}}}+\left|\mathrm{e}_{1}\right| \mathbf{n}_{E}^{\mathrm{e}_{1}}\right) / 2 \\
0 & \left(\left|\mathrm{e}_{1}\right| \mathbf{n}_{E}^{\mathrm{e}_{1}}+\left|\mathrm{e}_{2}\right| \mathbf{n}_{E}^{\mathrm{e}_{2}}\right) / 2 \\
0 & \left(\left|\mathrm{e}_{2}\right| \mathbf{n}_{E}^{\mathrm{e}_{2}}+\left|\mathrm{e}_{3}\right| \mathbf{n}_{E}^{\mathrm{e}_{3}}\right) / 2 \\
\vdots & \vdots \\
0 & \left(\left|\mathrm{e}_{k_{E}-1}\right| \mathbf{n}_{E}^{\mathrm{e}_{k_{E}}-1}+\left|\mathrm{e}_{k_{E}}\right| \mathbf{n}_{E}^{\mathrm{e}_{E}}\right) / 2
\end{array}\right) \\
& =\left(\begin{array}{ccc}
0 & \left(y_{2}-y_{k_{E}}\right) / 2 & \left(x_{k_{E}}-x_{2}\right) / 2 \\
0 & \left(y_{3}-y_{1}\right) / 2 & \left(x_{1}-x_{3}\right) / 2 \\
0 & \left(y_{4}-y_{2}\right) / 2 & \left(x_{2}-x_{4}\right) / 2 \\
\vdots & \vdots & \vdots \\
0 & \left(y_{1}-y_{k_{E}-1}\right) / 2 & \left(x_{k_{E}-1}-x_{1}\right) / 2
\end{array}\right) .
\end{aligned}
$$

Due to the above construction the consistency condition can be written as

$$
\mathrm{MN}=\mathrm{R},
$$

where the matrices N, R are given in (5.1) and (5.2), respectively. Moreover, it is easy to check that

$$
\left(\mathrm{R}^{T} \mathrm{~N}\right)(i, j)=\left(\mathrm{N}^{T} \mathrm{MN}\right)(i, j)=\int_{E} \nabla \rho_{i} \cdot \nabla \rho_{j} \mathrm{~d} x=: \mathrm{K}(i, j) \quad i, j=1,2,3,
$$

with $\mathrm{K}(i, j)$ clearly equal to $|E|$ if $i=j=2$ or $i=j=3$ and zero otherwise, that is,

$$
\mathrm{R}^{T} \mathrm{~N}=\left(\begin{array}{ccc}
0 & 0 & 0 \\
0 & |E| & 0 \\
0 & 0 & |E|
\end{array}\right)
$$

Equivalence (5.5) can be checked also taking into account the algebraic expressions of N, R given in (5.1) and (5.2), respectively. Indeed, we have

$$
\mathrm{R}^{T} \mathrm{~N}=\left(\begin{array}{ccc}
0 & 0 & 0 \\
0 & \frac{1}{2} \sum_{i=1}^{k_{E}}\left(x_{i} y_{i+1}-x_{i+1} y_{i}\right) & 0 \\
0 & 0 & \frac{1}{2} \sum_{i=1}^{k_{E}}\left(x_{i} y_{i+1}-x_{i+1} y_{i}\right)
\end{array}\right)
$$


which is indeed (5.5), taking into account the shoelace formula, according to which the area of the polygon $E$ (with sign) is given by

$$
\frac{1}{2} \sum_{i=1}^{k_{E}}\left(x_{i} y_{i+1}-x_{i+1} y_{i}\right) \text {. }
$$

Finally, the matrix $M$ is built as follows. Let

$$
\mathrm{P}=\mathrm{I}-\mathrm{N}\left(\mathrm{N}^{T} \mathrm{~N}\right)^{-1} \mathrm{~N}^{T}
$$

with I the $k_{E} \times k_{E}$ identity matrix. The matrix $\mathrm{P}$ is the projection matrix on the space orthogonal to the columns of $\mathrm{N}$. Then, we set

$$
\mathrm{M}=\frac{1}{|E|} \mathrm{RR}^{T}+s \mathrm{P},
$$

with $s=\operatorname{trace}\left(\frac{1}{|E|} \mathrm{RR}^{T}\right)>0$ a scaling factor. Recalling (5.4) and the definition of $\mathrm{P}$, it is easy to check that the above matrix $M$ satisfies the consistency condition (5.3). Moreover, also the stability property (S2) can be proved; see for instance [9, 3].

We remark that, whenever the mesh is made of triangles, the matrix $\mathrm{M}$ coincides with the (elemental) finite element stiffness matrix, i.e., on triangular elements the MFD method and FEM are the same. Indeed, on the one hand, the projection matrix $P$ turns out to be the null matrix, and the matrix $M$ becomes:

$$
\mathrm{M}=\frac{1}{|E|} \mathrm{RR}^{T}=\frac{1}{4|E|}\left(\begin{array}{lll}
m_{11} & m_{12} & m_{13} \\
m_{21} & m_{22} & m_{23} \\
m_{31} & m_{32} & m_{33}
\end{array}\right),
$$

where

$$
\begin{aligned}
m_{11} & =\left(x_{2}-x_{3}\right)^{2}+\left(y_{2}-y_{3}\right)^{2}, \\
m_{12}=m_{21} & =-\left(x_{1}-x_{3}\right)\left(x_{2}-x_{3}\right)-\left(y_{1}-y_{3}\right)\left(y_{2}-y_{3}\right), \\
m_{13}=m_{31} & =\left(x_{1}-x_{2}\right)\left(x_{2}-x_{3}\right)+\left(y_{1}-y_{2}\right)\left(y_{2}-y_{3}\right), \\
m_{22} & =\left(x_{1}-x_{3}\right)^{2}+\left(y_{1}-y_{3}\right)^{2}, \\
m_{23}=r_{32} & =-\left(x_{1}-x_{2}\right)\left(x_{1}-x_{3}\right)-\left(y_{1}-y_{2}\right)\left(y_{1}-y_{3}\right), \\
m_{33} & =\left(x_{1}-x_{2}\right)^{2}+\left(y_{1}-y_{2}\right)^{2} .
\end{aligned}
$$

On the other hand, we recall that the Lagrangian finite element shape functions $\varphi_{i}(x, y), i=1,2,3$, can be written as

$$
\begin{aligned}
& \varphi_{1}(x, y)=\frac{1}{2|E|}\left[\left(x_{2} y_{3}-x_{3} y_{2}\right)+\left(y_{2}-y_{3}\right) x+\left(x_{3}-x_{2}\right) y\right], \\
& \varphi_{2}(x, y)=\frac{1}{2|E|}\left[\left(x_{3} y_{1}-x_{1} y_{3}\right)+\left(y_{3}-y_{1}\right) x+\left(x_{1}-x_{3}\right) y\right], \\
& \varphi_{3}(x, y)=\frac{1}{2|E|}\left[\left(x_{1} y_{2}-x_{2} y_{1}\right)+\left(y_{1}-y_{2}\right) x+\left(x_{2}-x_{1}\right) y\right],
\end{aligned}
$$


and therefore

$$
\begin{aligned}
& \nabla \varphi_{1}(x, y)=\frac{1}{2|E|}\left(y_{2}-y_{3}, x_{3}-x_{2}\right), \\
& \nabla \varphi_{2}(x, y)=\frac{1}{2|E|}\left(y_{3}-y_{1}, x_{1}-x_{3}\right), \\
& \nabla \varphi_{3}(x, y)=\frac{1}{2|E|}\left(y_{1}-y_{2}, x_{2}-x_{1}\right) .
\end{aligned}
$$

Therefore, a straightforward calculation shows that the stiffness matrix $\mathrm{V}$ associated to the Lagrangian finite element shape functions has components

$$
\mathrm{V}(i, j):=\int_{E} \nabla \varphi_{j} \cdot \nabla \varphi_{i} \mathrm{~d} x=|E|\left(\nabla \varphi_{i}\right)^{T} \cdot\left(\nabla \varphi_{j}\right)=\mathrm{M}(i, j), \quad i, j=1,2,3
$$

\section{NumericAl RESUlts}

This section is devoted to present some numerical computations to confirm the theoretical results of the previous sections.

We consider the domain $\Omega=]-1,1\left[{ }^{2}\right.$. For a parameter $0<r<1$, we define the (continuous) load

$$
f(x, y):= \begin{cases}-8\left(2 x^{2}+2 y^{2}-r^{2}\right) & \text { if } \sqrt{x^{2}+y^{2}}>r \\ -8 r^{2}\left(1-x^{2}-y^{2}+r^{2}\right) & \text { if } \sqrt{x^{2}+y^{2}} \leq r\end{cases}
$$

and the Dirichlet boundary data $g(x, y):=\left(x^{2}+y^{2}-r^{2}\right)^{2}$. We consider a constant obstacle $\psi(x, y):=0$, so that the exact minimizer of model problem (2.1) is given by

$$
u(x, y):=\left(\max \left\{x^{2}+y^{2}-r^{2}, 0\right\}\right)^{2}
$$

cf. [26]. Figure 1 (left) depicts the minimizer $u$ together with the obstacle $\psi$ in the case $r=0.7$. The obstacle problem has been solved numerically by the Projected Successive Over Relaxation (PSOR) method [16, 18, 21]. More precisely, we discretized the corresponding unconstrained problem (that is, the Poisson equation) by means of MFD method which reads in matrix form as $A \widetilde{u}_{h}=f$. Then, $A$ is decomposed as $A=D+L+U$ for the projected Gauss-Seidel successive overrelaxation iteration (with over-relaxation parameter $\omega$ ), and the minimizer $u_{h}$ is found by constrained iteration up to a user-defined tolerance TOL. The initial guess is $\max \left\{\widetilde{u}_{h}, \psi\right\}$ where $\widetilde{u}_{h}$ is the solution to the unconstrained problem $\mathrm{A} \widetilde{u}_{h}=\mathrm{f}$. We refer to [18, 21] for more details. Throughout the section, the over-relaxation parameter $\omega$ has been chosen as $\omega=1.75$, and the tolerance TOL in the iterative scheme is fixed equal to $10^{-9}$.

We tested four different sequences of decompositions, that we denote by triangular, quadrilateral, median-type 1 and median-type 2. An example of two consecutive levels of all the considered decomposition is shown in Figure 2 An example of MFD minimizer together with the obstacle $\psi$ on a median-type 1 polygonal mesh is shown in Figure 1 (right). 

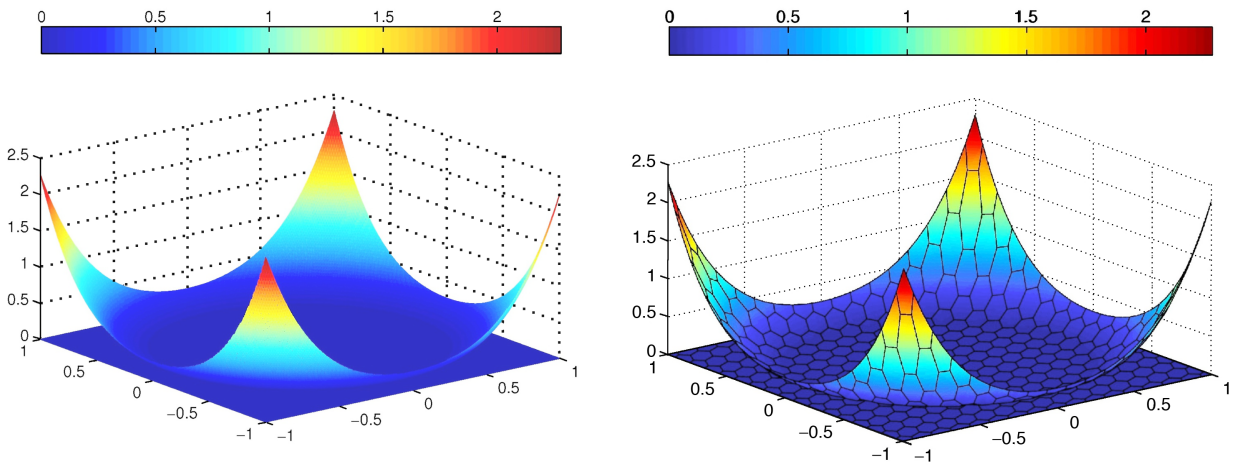

Figure 1. Left: exact minimizer $u$ with the obstacle $\psi(r=0.7)$. Right: MFD minimizer $u_{h}$ with the obstacle $\psi(r=0.7)$.
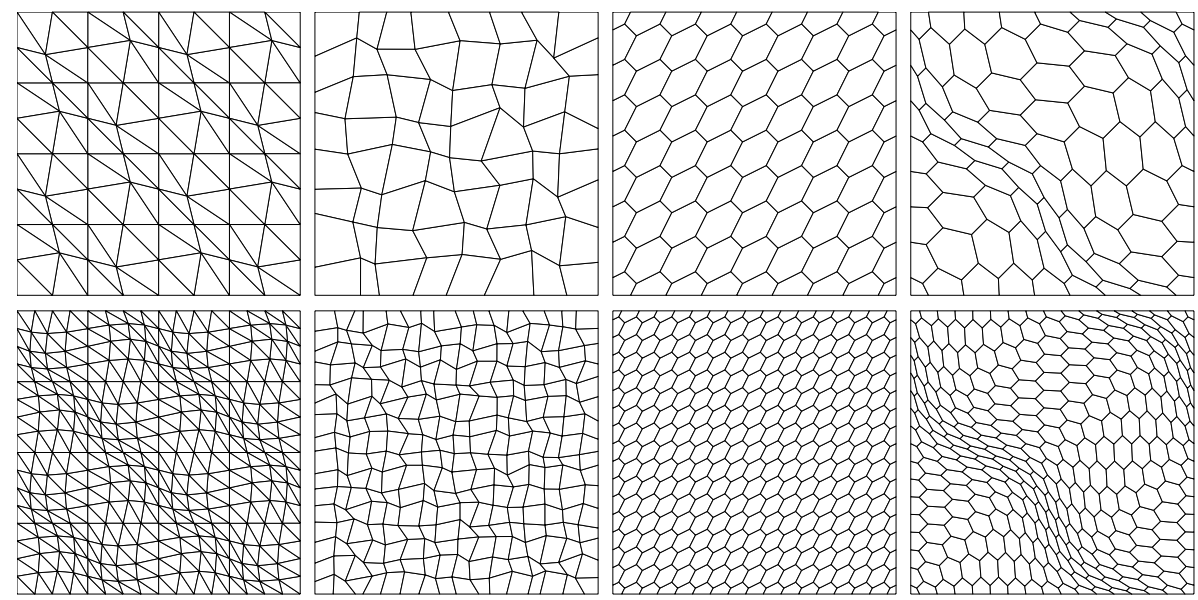

Figure 2. Two samples of the considered decompositions of $\Omega=$ ]$-1,1[2$ : one coarser (top) and one finer (bottom). From left to right: triangular mesh, quadrilateral mesh and median-type 1, median-type 2 polygonal meshes.

In Table 1 we report the computed (relative) errors $\varepsilon_{1, h}^{r}\left(u^{I}, u_{h}\right)$ in the discrete energy norm defined in (3.2), i.e.,

$$
\varepsilon_{1, h}^{r}\left(u^{I}, u_{h}\right)=\frac{\left\|u^{I}-u_{h}\right\|_{1, h}}{\left\|u^{I}\right\|_{1, h}},
$$

for the sequence of triangular and quadrilateral decompositions. Here and in the following, $n_{P}$ denotes the number of polygons of the decomposition. In the last row of Table 1 we also report the computed convergence rates obtained by the linear regression algorithm. We can observe that on quadrilateral meshes the computed convergence rate is linear as predicted by Theorem 4.1 whereas on triangular decomposition convergence is achieved slightly better than expected; such a behaviour has been already observed in [3. The analogous results obtained on median-type 1 and median-type 2 decompositions are shown in Figure 3 (loglog scale), and are 
TABLE 1. Computed relative errors $\varepsilon_{1, h}^{r}\left(u^{I}, u_{h}\right)$ on the sequence of triangular and quadrilateral meshes.

\begin{tabular}{rrrr}
\hline \multicolumn{2}{c}{ triangular meshes } & \multicolumn{2}{c}{ quadrilateral meshes } \\
\hline$n_{P}$ & $\varepsilon_{1, h}^{r}\left(u^{I}, u_{h}\right)$ & \multicolumn{1}{c}{$n_{P}$} & $\varepsilon_{1, h}^{r}\left(u^{I}, u_{h}\right)$ \\
\hline 128 & $3.7452 \mathrm{e}-02$ & 64 & $6.4114 \mathrm{e}-02$ \\
512 & $1.1865 \mathrm{e}-02$ & 256 & $2.5172 \mathrm{e}-02$ \\
2048 & $3.4448 \mathrm{e}-03$ & 1024 & $1.2802 \mathrm{e}-02$ \\
8192 & $9.5227 \mathrm{e}-04$ & 4096 & $6.7499 \mathrm{e}-03$ \\
32768 & $2.7586 \mathrm{e}-04$ & 16384 & $3.4652 \mathrm{e}-03$ \\
\hline rate & 1.7809 & & 1.0318 \\
\hline
\end{tabular}

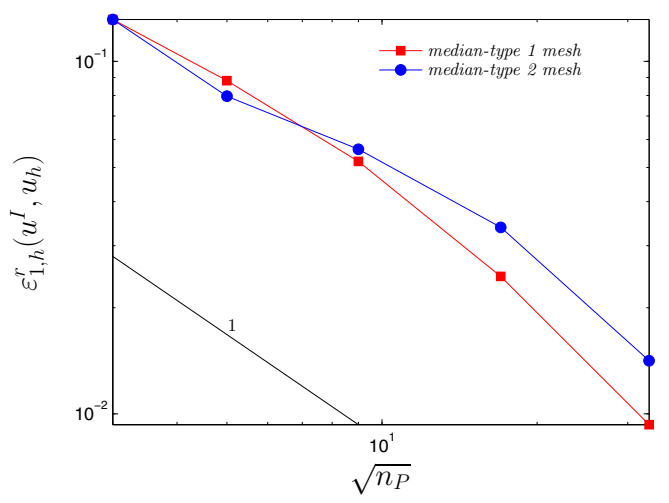

Figure 3. Computed relative errors $\varepsilon_{1, h}^{r}\left(u^{I}, u_{h}\right)$ versus the square root of the number of cells (loglog scale): median-type 1 and median-type 2 meshes.

indeed in agreement with our theoretical estimates. Next, we also investigate the (relative) error behaviour in the discrete $L^{2}$ - and $L^{\infty}$-type norms defined in (3.3). To this aim, we set

$$
\varepsilon_{0, h}^{r}\left(u^{I}, u_{h}\right):=\frac{\left\|u^{I}-u_{h}\right\|_{0, h}}{\left\|u^{I}\right\|_{0, h}}, \quad \varepsilon_{\infty, h}^{r}\left(u^{I}, u_{h}\right):=\frac{\left\|u^{I}-u_{h}\right\|_{\infty, h}}{\left\|u^{I}\right\|_{\infty, h}} .
$$

The computed errors $\varepsilon_{0, h}^{r}\left(u^{I}, u_{h}\right)$ and $\varepsilon_{\infty, h}^{r}\left(u^{I}, u_{h}\right)$ versus the square root of the number of cells are reported in Figure 4 (loglog scale). Results reported in Figure 4(a) refer to triangular and quadrilateral meshes, whereas results obtained on median-type 1 and median-type 2 decompositions are shown in Figure 4(b). A quadratic convergence rate is clearly observed.

Next, we compare the performance of MFD (restricted to triangular meshes) and FEM, and we investigate the effects of employing the discrete norms (3.2) and (3.3) instead of their continuous counterparts (that is, the $H^{1}(\Omega)$ and $L^{2}(\Omega)$ norms). First, we employ the FEM to approximate the model problem under consideration 
(a) Triangular and quadrilateral meshes.
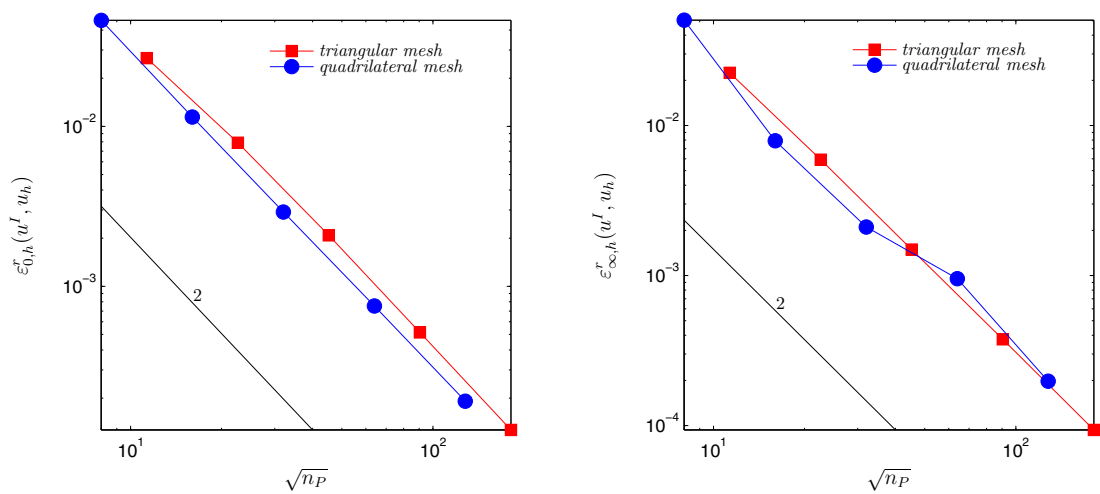

(b) Median meshes.
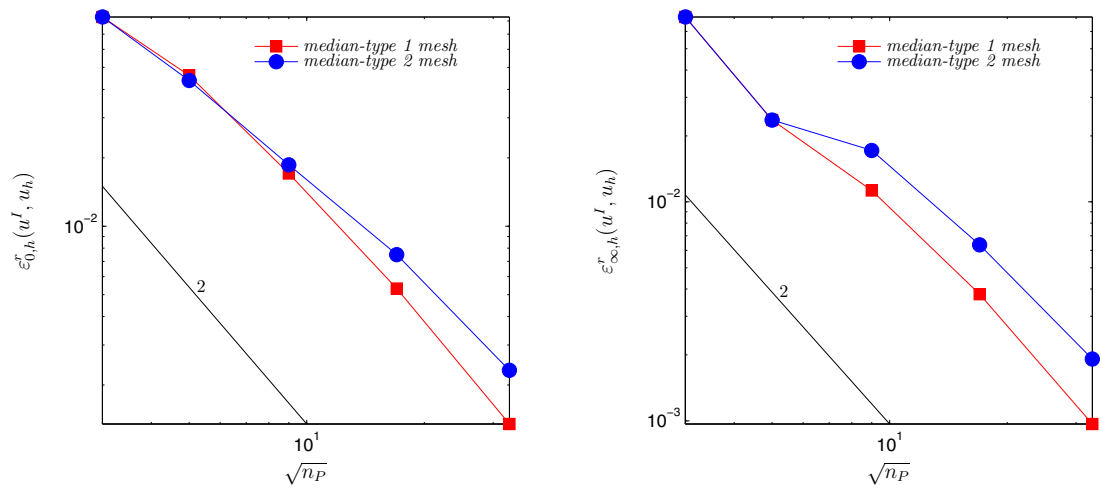

Figure 4. Computed relative errors $\varepsilon_{0, h}^{r}\left(u^{I}, u_{h}\right)$ (left) and $\varepsilon_{\infty, h}^{r}\left(u^{I}, u_{h}\right)$ (right) versus the square root of the number of cells (loglog scale).

on a sequence of triangular decompositions, and compute the (relative) errors both in the $H^{1}(\Omega)$ seminorm and in the $L^{2}(\Omega)$ norm

$$
\varepsilon_{1}^{r}\left(u^{I}, u_{h}\right):=\frac{\left|u^{I}-u_{h}\right|_{H^{1}(\Omega)}}{\left|u^{I}\right|_{H^{1}(\Omega)}}, \quad \varepsilon_{0}^{r}\left(u^{I}, u_{h}\right):=\frac{\left\|u^{I}-u_{h}\right\|_{L^{2}(\Omega)}}{\left\|u^{I}\right\|_{L^{2}(\Omega)}} .
$$

To compute the right-hand side of the finite element variational formulation, we have employed the barycenter quadrature formula, which is exact for linear polynomials, and therefore it is consistent with the quadrature formula (3.7). Note that the right-hand side is the only difference between FEM and MFD in the triangular case, since the stiffness matrix comes out to be always the same. In Table 2 we compare the finite element relative errors computed as in (6.3) with their discrete counterpart, namely $\varepsilon_{1, h}^{r}\left(u^{I}, u_{h}\right)$ and $\varepsilon_{0, h}^{r}\left(u^{I}, u_{h}\right)$. We clearly observe that, for both $s=0$ and $s=1$, the relative error $\varepsilon_{s, h}^{r}\left(u^{I}, u_{h}\right)$ (which only employs nodal values) is systematically smaller than $\varepsilon_{s}^{r}\left(u^{I}, u_{h}\right)$. This phenomenon is probably related to an improved accuracy in the nodal value approximation. Next, we compare results 
obtained by MFD and FEM. In Figure 5 we plot the computed errors $\varepsilon_{s, h}^{r}\left(u^{I}, u_{h}\right)$, $s=0,1$, versus the square root of the number of cells.

TABLE 2. Finite element approximation: comparison between discrete and continuous norms, namely $\varepsilon_{s, h}^{r}\left(u^{I}, u_{h}\right)$ and $\varepsilon_{s}^{r}\left(u^{I}, u_{h}\right)$, $s=0,1$.

\begin{tabular}{lrrrr}
\hline$n_{P}$ & $\varepsilon_{1}^{r}\left(u^{I}, u_{h}\right)$ & $\varepsilon_{1, h}^{r}\left(u^{I}, u_{h}\right)$ & $\varepsilon_{0}^{r}\left(u^{I}, u_{h}\right)$ & $\varepsilon_{0, h}^{r}\left(u^{I}, u_{h}\right)$ \\
\hline 128 & $2.4692 \mathrm{e}-01$ & $1.7761 \mathrm{e}-02$ & $2.0321 \mathrm{e}-01$ & $1.1906 \mathrm{e}-02$ \\
512 & $1.2123 \mathrm{e}-01$ & $7.8972 \mathrm{e}-03$ & $4.8759 \mathrm{e}-02$ & $4.6443 \mathrm{e}-03$ \\
2048 & $6.0299 \mathrm{e}-02$ & $2.3997 \mathrm{e}-03$ & $1.2071 \mathrm{e}-02$ & $1.2427 \mathrm{e}-03$ \\
8192 & $3.0113 \mathrm{e}-02$ & $7.3530 \mathrm{e}-04$ & $3.0086 \mathrm{e}-03$ & $3.1091 \mathrm{e}-04$ \\
32768 & $1.5052 \mathrm{e}-02$ & $2.3510 \mathrm{e}-04$ & $7.5225 \mathrm{e}-04$ & $7.7263 \mathrm{e}-05$ \\
\hline rate & 1.0081 & 1.5903 & 2.0174 & 1.8436 \\
\hline
\end{tabular}

(a) $\varepsilon_{1, h}^{r}\left(u^{I}, u_{h}\right)$

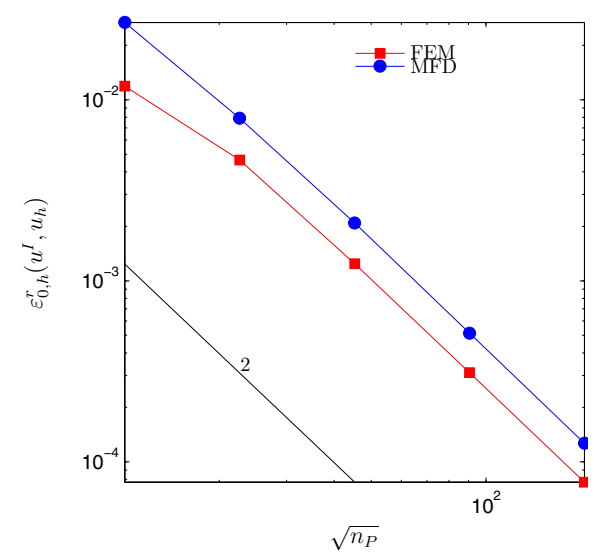

(b) $\varepsilon_{0, h}^{r}\left(u^{I}, u_{h}\right)$

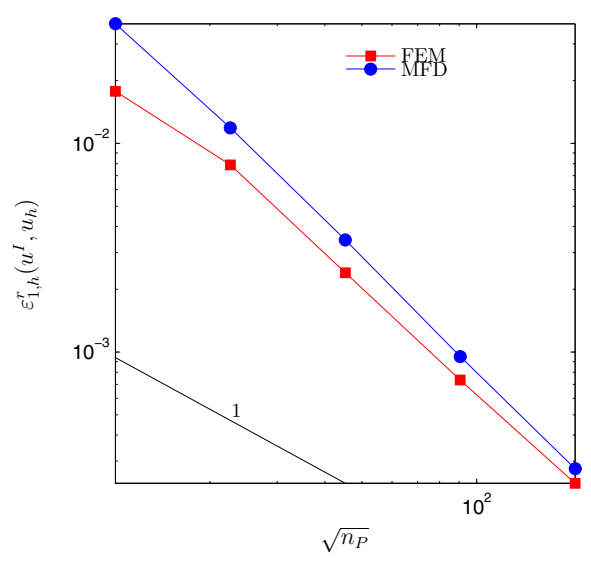

Figure 5. Comparison between FEM and MFD: computed relative errors $\varepsilon_{s, h}^{r}\left(u^{I}, u_{h}\right), s=0,1$, versus the square root of the number of cells (loglog scale).

We observe that both MFD and FEM achieve asymptotic convergence at a moderately better rate than predicted by our theoretical estimates, and that MFD produces a slightly larger error.

Finally, we present some numerical computations to confirm that the theoretical results of the previous sections are valid also on nonconvex domains (cf. Remark 4.3). To this aim we choose the L-shaped domain $\Omega=]-1,1\left[2 \backslash[0,1]^{2}\right.$, and we consider the same test problem as before. Figure 6 (right) shows a plot of the MFD minimizer $u_{h}$ together with the obstacle $\psi(r=0.7)$. We tested the MFD method on a sequence of quadrilateral meshes: a sample is shown in Figure 6 (left). 
The computed relative errors $\varepsilon_{1, h}^{r}\left(u^{I}, u_{h}\right)$ and $\varepsilon_{0, h}^{r}\left(u^{I}, u_{h}\right)$ are reported in Figure 7 (loglog scale): as predicted by Theorem 4.1, we observe a linear convergence rate in the discrete energy norm. We also observe that the relative error in the discrete $L^{2}$ norm tends to zero quadratically as the mesh is refined.
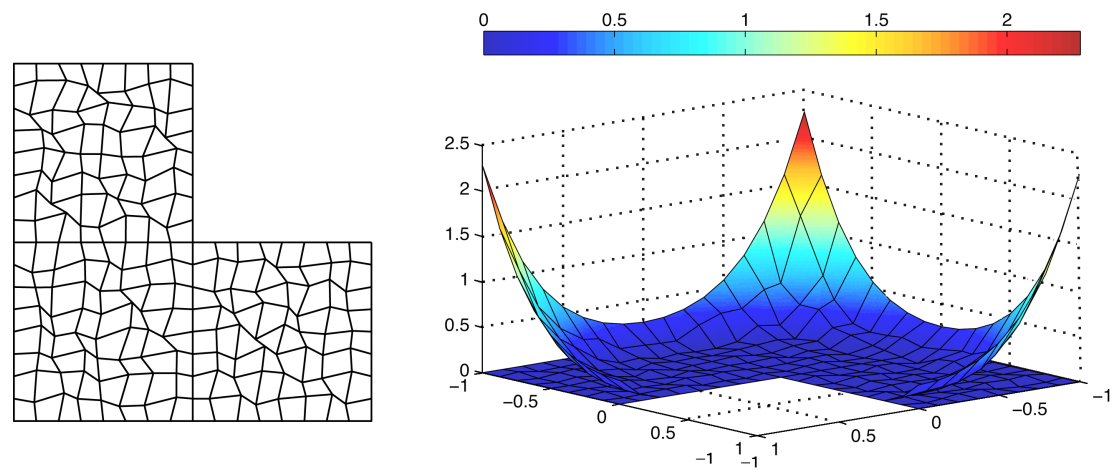

FiguRE 6. L-shaped domain: a sample of the quadrilateral decomposition (left), and the corresponding MFD minimizer $u_{h}$ together with the obstacle $\psi$ (right).

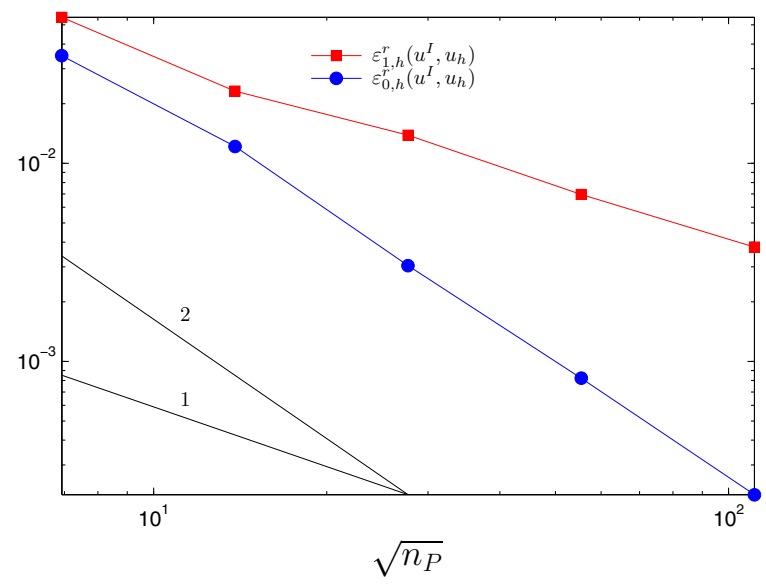

FIGURE 7. L-shaped domain: computed relative errors $\varepsilon_{1, h}^{r}\left(u^{I}, u_{h}\right)$ and $\varepsilon_{0, h}^{r}\left(u^{I}, u_{h}\right)$ versus the square root of the number of cells (loglog scale).

\section{Appendix A. Proof of M4}

Accordingly to $(\mathrm{H} 1)-(\mathrm{H} 2)$, the polyhedron $E$ may be nonstar-shaped. Therefore the approximation bound in (M4) must rely on the more general results in [17]. In order to show that, given our assumptions $(\mathrm{H} 1)-(\mathrm{H} 2)$, the constant appearing in (M4) is uniformly bounded, we derive here explicitly the proof. In the following steps we will use the symbol $H^{0}$ for the $L^{2}$ space. Moreover, for simplicity of 


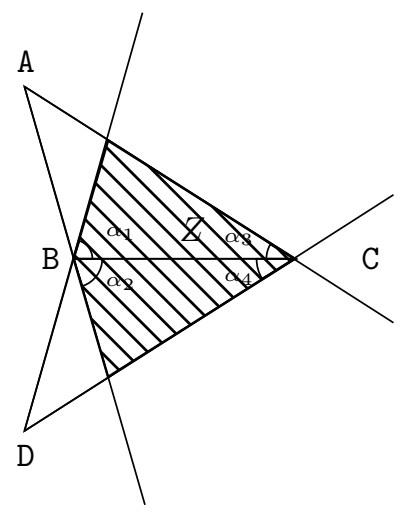

FiguRE 8 . The region $Z$ indicates the intersection of the two cones built by prolungating the edges of the triangles $\mathrm{ABC}$ and $\mathrm{DBC}$.

notation, we will indicate with the symbol $A \cup B$ the internal part of the union of the closures of any open sets $A$ and $B$.

We start with the following result.

Lemma A.1. Let $k$ and $m$ be nonnegative integer numbers. Let $\omega_{h}$ be a conforming connected mesh of $N$ shape regular triangles satisfying the regularity condition $(\mathrm{H} 2)$ and $\omega=\bigcup_{T \in \omega_{h}} T$. Then, there exists a constant $C^{\prime}=C^{\prime}(\rho, N, m, k)$ such that for all polynomials $v^{(m)}$ of degree at most $m$ on $\omega$,

$$
\left|v^{(m)}\right|_{H^{k}(\omega)} \leq C^{\prime}\left|v^{(m)}\right|_{H^{k}(T)} \quad \forall T \in \omega_{h} .
$$

Proof. We only sketch the proof. Given $N$, there exist a finite number of possible connectivity configurations of the triangles in $\omega_{h}$. Thus, there exist a finite number of reference meshes $\widehat{\omega}_{h}$ such that each admissible mesh $\omega_{h}$ can be mapped into a reference mesh. We now note that the two norms appearing in (A.1) have the same kernel. Therefore, since the space of polynomials of degree $m$ is finite dimensional, there exists a constant $C^{\prime \prime}=C^{\prime \prime}(N, m, k)$ such that the lemma is true on all reference meshes. Finally, the proof of the lemma follows easily from a standard scaling argument, also observing that all the involved maps have bounded norms due to the mesh regularity of $\omega_{h}$.

We are now able to show the proof of (M4). Given $k, m \in \mathbb{N}$ nonnegative integers and $s \in \mathbb{R}, 0 \leq s \leq m$, we consider any element $E \in \Omega_{h}$. We will prove property (M4) by induction on the number $N$ of triangles in $\left.\mathcal{T}_{h}\right|_{E}$. We observe that such number $N$ is bounded by $N_{s}$ of Assumption ( $\left.\mathrm{H} 1\right)$.

We start observing that, if $T_{1}$ and $T_{2}$ are two triangles (of a shape regular family of meshes) which share one edge, then the union $U=T_{1} \cup T_{2}$ is star shaped with respect to a ball. Moreover, the ratio of the radius of such ball divided by the diameter of $U$ is uniformly bounded from below. Indeed, the following simple proof applies. Given any two triangles $\mathrm{ABC}$ and $\mathrm{DBC}$ as in Figure 8 , let $Z$ indicate the quadrilateral obtained by the intersection of the two cones built by edge prolungation, i.e., the shaded region in Figure 8. Clearly, the region $U$ given by the union of the two triangles is star shaped with respect to $Z$. Moreover, due to the shape 
regularity of the mesh, all the angles $\alpha_{1}, \ldots, \alpha_{4}$ in Figure 8 are uniformly bounded from below. Therefore, it is easy to check that there exists a ball in $Z$ with radius $R \geq \theta|\mathrm{BC}|$, with $|\mathrm{BC}|$ length of the edge $\mathrm{BC}$ and the real $\theta>0$ uniformly bounded from below. This proves the assertion.

As a consequence of the above result, for any $U=T_{1} \cup T_{2}$ we can apply the wellknown polynomial approximation bound on star-shaped domains; see for instance [17. For all $v \in H^{s+1}(U)$, there exists a polynomial of degree $m$, denoted by $v^{(m)}$, such that

$$
h_{U}^{k}\left|v-v^{(m)}\right|_{H^{k}(U)} \leq C_{U} h_{U}^{s+1}|v|_{H^{s+1}(U)},
$$

with $C_{U}=C_{U}(k, m, s, \rho)$ and where $\rho$ is the shape regularity constant appearing in $(\mathrm{H} 2)$. Furthermore, the same result obviously applies if $U$ is a single triangle. Thus we obtained that, if $N=1$ or 2 , the bound in (M4) is proved with $C_{a p p}=C_{U}$.

We now assume that such a bound holds true for any mesh of up to $N$ triangles (with a constant $C$ depending only on $k, m, s, \rho$, and $N$ ). We want to show that it holds also for any mesh $\left.\mathcal{T}_{h}\right|_{E}$ composed of $N+1$ triangles. Therefore, let $T_{1}$ be any triangle from $\left.\mathcal{T}_{h}\right|_{E}$ and let $T_{2}$ be any other triangle from $\left.\mathcal{T}_{h}\right|_{E}$ which has a common face with $T_{1}$. We introduce the following two subsets of $E$ :

$$
A=T_{1} \cup T_{2} \quad \text { and } \quad B=\left\{\bigcup T:\left.T \in \mathcal{T}_{h}\right|_{E}, T \neq T_{1}\right\} .
$$

Clearly, there hold $A \cup B=E$ and $A \cap B=T_{2}$. Due to the induction hypothesis, the interpolation bound in (M4) is satisfied for both sets $A$ and $B$. Given any $v \in H^{s+1}(E)$, we denote with $v_{A}^{(m)}$ and $v_{B}^{(m)}$ the interpolation polynomials (of degree $m$ ) for $v$ on the two subsets $A$ and $B$, respectively. We thus have

$$
\begin{aligned}
& h_{A}^{k}\left|v-v_{A}^{(m)}\right|_{H^{k}(A)} \leq C_{A} h_{A}^{s+1}|v|_{H^{s+1}(A)}, \\
& h_{B}^{k}\left|v-v_{B}^{(m)}\right|_{H^{k}(B)} \leq C_{B} h_{B}^{s+1}|v|_{H^{s+1}(B)} .
\end{aligned}
$$

Making use of property (M2), we easily obtain from the bounds in (A.3)

$$
\begin{aligned}
& h_{E}^{k}\left|v-v_{A}^{(m)}\right|_{H^{k}(A)} \leq C_{A} h_{E}^{s+1}|v|_{H^{s+1}(A)}, \\
& h_{E}^{k}\left|v-v_{B}^{(m)}\right|_{H^{k}(B)} \leq C_{B} h_{E}^{s+1}|v|_{H^{s+1}(B)}
\end{aligned}
$$

with different constants $C_{A}$ and $C_{B}$, but still depending only on $k, m, s, \rho$, and $N_{s}$. Now the triangle inequality yields

$$
\left|v-v_{A}^{(m)}\right|_{H^{k}(E)} \leq\left|v-v_{A}^{(m)}\right|_{H^{k}(A)}+\left|v-v_{B}^{(m)}\right|_{H^{k}(B)}+\left|v_{A}^{(m)}-v_{B}^{(m)}\right|_{H^{k}(B)} .
$$

For the last term in the above equation, we apply Lemma A.1 (with $\omega=B$ and $T=T_{2}$ ) and the triangle inequality to derive

$$
\begin{aligned}
\left|v_{A}^{(m)}-v_{B}^{(m)}\right|_{H^{k}(B)} & \leq C^{\prime}\left|v_{A}^{(m)}-v_{B}^{(m)}\right|_{H^{k}\left(T_{2}\right)} \\
& \leq C^{\prime}\left(\left|v_{A}^{(m)}-v\right|_{H^{k}\left(T_{2}\right)}+\left|v-v_{B}^{(m)}\right|_{H^{k}\left(T_{2}\right)}\right) \\
& \leq C^{\prime}\left(\left|v_{A}^{(m)}-v\right|_{H^{k}(A)}+\left|v-v_{B}^{(m)}\right|_{H^{k}(B)}\right) .
\end{aligned}
$$

We finally combine (A.5), (A.6) and the interpolation bounds (A.4). This yields

$$
h_{E}^{k}\left|v-v_{A}^{(m)}\right|_{H^{k}(E)} \leq \sqrt{2}\left(1+C^{\prime}\right) \max \left\{C_{A}, C_{B}\right\} h_{E}^{s+1}|v|_{H^{s+1}(E)} .
$$

Therefore property (M4) is proved. 


\section{ACKNOWLEDGMENTS}

We are grateful to Gianmarco Manzini for the help in generating polygonal median meshes.

\section{REFERENCES}

1. P.F. Antonietti, L. Beirão da Veiga, and M. Verani, A mimetic discretization of elliptic obstacle problems, Tech. report, MOX, Dipartimento di Matematica, Politecnico di Milano, 2010, http://mox.polimi.it/it/progetti/pubblicazioni/.

2. L. Beirão da Veiga, A mimetic finite difference method for linear elasticity, 2010, M2AN Math. Model. Numer. Anal., 44 (2010), no. 2, 231-250. doi 10.1051/m2an/2010001. MR2655949 (2011d:65317)

3. L. Beirão da Veiga, V. Gyrya, K. Lipnikov, and G. Manzini, Mimetic finite difference method for the Stokes problem on polygonal meshes, J. Comput. Phys 228 (2009), 72157232. MR2568590 (2010k:65229)

4. L. Beirão da Veiga, K. Lipnikov, and G. Manzini, Convergence analysis of the high-order mimetic finite difference method, Numer. Math. 113 (2009), no. 3, 325-356. MR2534128 (2010g:65180)

5. L. Beirão da Veiga and G. Manzini, An a posteriori error estimator for the mimetic finite difference approximation of elliptic problems, Int. J. Numer. Methods Engrg. 76 (2008), no. 11, 1696-1723. MR2468392 (2009j:65294)

6. L. Beirão da Veiga and D. Mora, A mimetic discretization of the Reissner-Mindlin plate bending problem, Numerische Mathematik 117 (2011), 425-462, 10.1007/s00211-010-0358-8. MR2772415 (2012b:65174)

7. H. Brezis, Problèmes unilatéraux, J. Math. Pures. Appl. 51 (1972), no. 9, 1-168. MR0428137 $(55: 1166)$

8. H. Brezis and G. Stampacchia, Sur la régularité de la solution d'inéquations elliptiques, Bull. Soc. Math. France 96 (1968), 153-180. MR0239302 (39:659)

9. F. Brezzi, A. Buffa, and K. Lipnikov, Mimetic finite differences for elliptic problems, M2AN Math. Model. Numer. Anal. 43 (2009), no. 2, 277-295. MR2512497 (2010d:65285)

10. F. Brezzi, W.W. Hager, and P.A. Raviart, Error estimates for the finite element solution of variational inequalities, Numer. Math. 28 (1977), 431-443. MR0448949 (56:7254)

11. F. Brezzi, K. Lipnikov, and M. Shashkov, Convergence of the mimetic finite difference method for diffusion problems on polyhedral meshes, SIAM J. Numer. Anal. 43 (2005), no. 5, 18721896. MR2192322 (2006j:65311)

12. F. Brezzi, K. Lipnikov, M Shashkov, and V. Simoncini, A new discretization methodology for diffusion problems on generalized polyhedral meshes, Comput. Methods Appl. Mech. Engrg. 196 (2007), no. 37-40, 3682-3692. MR2339994 (2008f:65102)

13. F. Brezzi, K. Lipnikov, and V. Simoncini, A family of mimetic finite difference methods on polygonal and polyhedral meshes, Math. Models Methods Appl. Sci. 15 (2005), no. 10, 15331551. MR2168945 (2006i:65172)

14. A. Cangiani and G. Manzini, Flux reconstruction and pressure post-processing in mimetic finite difference methods, Comput. Methods Appl. Mech. Engrg. 197 (2008), no. 9-12, 933945. MR 2376968 (2009i:65190)

15. P. G. Ciarlet, The finite element method for elliptic problems, North-Holland, Amsterdam, 1978. MR0520174(58:25001)

16. C. W. Cryer, Successive overrelaxation methods for solving linear complementarity problems arising from free boundary problems, Free boundary problems, Vol. I (Pavia, 1979), Ist. Naz. Alta Mat. Francesco Severi, Rome, 1980, pp. 109-131. MR630716 (83c:65059)

17. T. Dupont and R. Scott, Polynomial approximation of functions in Sobolev spaces, Math. Comp. 34 (1980), no. 150, 441-463. MR.559195 (81h:65014)

18. C. M. Elliott and J. R. Ockendon, Weak and variational methods for moving boundary problems, Research Notes in Mathematics, vol. 59, Pitman (Advanced Publishing Program), Boston, Mass., 1982. MR650455 (83i:35157)

19. R. S. Falk, Error estimates for the approximation of a class of variational inequalities, Math. Comput. 28 (1974), 963-971. MR0391502 (52:12323) 
20. A. Friedman, Variational principles and free-boundary problems, Pure and Applied Mathematics, John Wiley \& Sons Inc., New York, 1982. MR679313 (84e:35153)

21. R. Glowinski, J.-L. Lions, and R. Trémolières, Numerical analysis of variational inequalities, Studies in Mathematics and its Applications, vol. 8, North-Holland Publishing Co., Amsterdam, 1981. MR635927 (83k:49014)

22. P. Jaillet, D. Lamberton, and B. Lapeyre, Variational inequalities and the pricing of American options, Acta Appl. Math. 21 (1990), no. 3, 263-289. MR.1096582 (92d:90013)

23. D. Kinderlehrer and G. Stampacchia, An introduction to variational inequalities and their applications, Classics in Applied Mathematics, vol. 31, Society for Industrial and Applied Mathematics (SIAM), Philadelphia, PA, 2000. MR.1786735 (2002d:49001)

24. K. Lipnikov, J.D. Moulton, and D. Svyatskiy, A Multilevel Multiscale Mimetic ( $\left.M^{3}\right)$ method for two-phase flows in porous media, J. Comp. Phys. 227 (2008), 6727-6753. MR.2435429 (2009f:76129)

25. K. Lipnikov, M. Shashkov, and I. Yotov, Local flux mimetic finite difference methods, Numer. Math. 112 (2009), no. 1, 115-152. MR2481532(2010b:65238)

26. R. H. Nochetto, K. G. Siebert, and A. Veeser, Pointwise a posteriori error control for elliptic obstacle problems, Numer. Math. 95 (2003), no. 1, 163-195. MR1993943 (2004g:49055)

27. J.-F. Rodrigues, Obstacle problems in mathematical physics, North-Holland Mathematics Studies, vol. 134, North-Holland Publishing Co., Amsterdam, 1987. MR880369 (88d:35006)

MOX, Dipartimento di Matematica, Politecnico di Milano, Piazza Leonardo da Vinci 32, I-20133 Milano, Italy

E-mail address: paola.antonietti@polimi.it

Dipartimento di Matematica, Università di Milano, Via Saldini 50, I-20133 Milano, ITALY

E-mail address: lourenco.beirao@unimi.it

MOX, Dipartimento di Matematica, Politecnico di Milano, Piazza Leonardo da Vinci 32, I-20133 Milano, Italy

E-mail address: marco.verani@polimi.it 